\title{
Optimal Pricing of Public Lotteries and Comparison of Competing Mechanisms
}

\author{
Chen Ling \\ School of Economics \\ Southwestern University of Finance and Economics \\ Chengdu, Sichuan 611130 China \\ lingchen@swufe.edu.cn \\ phone: +86-28-61331710 \\ David Scrogin* \\ Department of Economics \\ College of Business Administration \\ University of Central Florida \\ Orlando, FL 32816-1400 USA \\ dscrogin@bus.ucf.edu \\ phone: 407-823-4129
}

\begin{abstract}
The negative effects of price controls on consumer surplus in competitive markets are well known. But what of consumer surplus if supply is fixed, as with rival but otherwise non-excludable goods held in public trust? This paper establishes optimal pricing rules for rationing indivisible units of such goods by lottery or mixture of a lottery and auction. The solution to the pricing problem appears in classic inverse elasticity form that may be directly implemented. Analysis of a rich class of private value distributions indicates the optimal lottery yields sizable gains in expected consumer surplus over competitive pricing and zero pricing.
\end{abstract}

${ }^{*}$ Corresponding author. Earlier versions of the paper were presented at the University of Colorado Environmental and Resources Workshop and seminars at the University of Central Florida and Southwestern University of Finance and Economics. We are especially grateful to Michael Caputo and Shelby Gerking for helpful comments and discussion. All errors or omissions are our sole responsibility. 


\section{Optimal Pricing of Public Lotteries and Comparison of Competing Mechanisms}

\section{Introduction}

The negative effects of price controls on consumer surplus in competitive markets are well known. As noted by Glaeser and Luttmer (2003) and Davis and Kilian (2011) in analyses of housing rent controls and U.S. natural gas distribution prior to deregulation, when prices are fixed and shortages arise, a nonmarket mechanism such as a queue or lottery is needed to distribute the available units and some will invariably be awarded to individuals who value the good less than others. Price controls also discourage production, contracting the long run competitive equilibrium as resources transfer to higher-valued uses or sit idle. Simply, consumer surplus declines as fewer units of the good are inefficiently traded.

But what of consumer surplus when supply is fixed over trading periods? Barring windfall gains to incumbents, can a price control be expected to increase consumer surplus over the competitive level and if so, what price maximizes expected consumer surplus? These questions were formally addressed by Bulow and Klemperer (2012) when the quantity supplied is endogenous to the price and rationed by lottery. They demonstrated for a class of functions that consumer surplus will decline if supply is sufficiently price elastic relative to demand. The question of optimal price controls in competitive markets is then moot.

In settings where supply is independent of price, the effect of a price control depends solely upon conditional demand, so consumer surplus may well exceed that of competitive pricing. The reasoning why is straightforward: while lowering price from the clearing rate engages those who value the good by less and less and reduces the probability any individual entrant will be drawn, the surplus realized by every entrant if drawn necessarily rises and so then may the mean surplus over entrants. ${ }^{1}$ Publicly produced goods and otherwise non-excludable but rival goods held in public

Similarly, if the distribution of private values is relatively dense in the lower tail, a marginally positive price can exclude a sizable fraction of individuals, increasing the probability of being drawn and the expected total and net value of the allocation. 
trust are commonly fixed in supply and subject to price controls, and examples abound where the dilemma of distributing such goods is resolved by lottery. ${ }^{2}$

Analysis of public lotteries dates to Seneca (1970) and Mumy and Hanke (1975), who extended the benefit-cost framework for project evaluation to settings where outputs are under-priced. Work has since focused upon lottery design and performance relative to competing rationing methods (e.g., Boyce, 1994; Taylor et al., 2003; Che et al., 2012) and empirical estimation of the non-market value of lottery-rationed goods (e.g., Buschena et al., 2001; Scrogin, 2005). To date, public lotteries have been treated as costless to consumers or price has been fixed arbitrarily below the clearing rate. It follows that an open question with significant practical implications regards what price control, if any, maximizes expected net benefits.

In this paper we establish optimal pricing rules for rationing goods by lottery. In the next section the equivalence of the conditional distribution of consumer surplus and of expected revenues over uniformly priced lotteries is first established. Inverse elasticity pricing rules for maximizing expected consumer surplus are then derived, with price appearing as an entry fee incurred by all participants, or a user fee incurred by the subset who receive units, or a combination of the two. In section three the pricing rules and mean and variance of consumer surplus are derived for a rich class of value distributions, and the performance of the optimal lottery relative to alternative pricing arrangements is evaluated numerically. The model is extended in section four to the hybrid setting considered by Evans et al. (2009), whereby a portion of supply is distributed competitively and the remainder by lottery. Section five summarizes the findings and discusses implementation of the lotteries.

2

Federal cases include the Urban Homesteading Act proposed by President Bush following Hurricane Katrina, whereby parcels of public land would be awarded to low-income households through a zero-price lottery in return for a pledge to build a permanent residence (Bush, 2005), and the transition by the U.S. National Park Service from a queue with wait times exceeding ten years to an annual two-part price lottery for rationing recreational use of the Grand Canyon (NPS, 2006). As noted by Boyce (1994) and Evans et al. (2009), lotteries also have a long history at state and local levels for rationing goods and services, including school admission, public housing, and consumptive uses of public lands and waterways. 


\section{Optimal Lottery Pricing}

A variety of explanations have been put forth why lotteries may be favored over competing mechanisms for rationing goods to the public. Citing their fairness, in that individuals have a positive though not necessarily equal probability of being drawn, Kahneman et al. (1986) reported survey findings where respondents preferred lotteries over market prices for distributing particular types of consumer goods. ${ }^{3}$ Boyce (1994) also demonstrated that lotteries may be majority-preferred over queues and auctions if chosen by referendum, particularly as the relative scarcity of the good rises. Clearly, otherwise off-margin bidders in Vickery-type auctions would prefer an alternative means by which to acquire the good, such as a lottery or the stochastic pricing rules considered by Shogren et al. (2001).

Aside from fairness and majority-preferences, lotteries have been shown to be superior on grounds of efficiency. Taylor et al. (2003) and Kho et al. (2007) demonstrated that lotteries can capture more total value than queues, though price was fixed at zero in their analyses. And compared to competitive pricing, Che et al. (2012) demonstrated that lotteries can capture more total value if individuals are budget constrained and their private valuations are independent of wealth. This is because some units will be awarded to high-value low-wealth individuals, and if transferable, low-value low-wealth individuals who happen to receive units will be inclined to sell them in the secondary market. Condorelli (2012) also showed that lotteries can be relatively efficient when it is costly to screen individuals according to their private valuations.

In the following, lottery pricing rules for maximizing expected consumer surplus are derived, and the performance of the optimal lottery is compared to alternative pricing rules. We begin by assuming that $Q^{*}$ units of an indivisible and non-transferable good are to be distributed. There are $N$ risk-neutral potential recipients who possess unit demands for the good that are represented by their

Fairness in the allocation is consistent with the utilitarian objective of distributional equity, or specific egalitarianism, discussed by Tobin (1970), whereby certain classes of goods are allocated in a manner yielding greater social equality than market pricing. 
private valuations $v($.$) , assumed iid over the interval [0, \bar{v}]$ according to the cumulative distribution function $F(v)$. The lottery proceeds in two stages. In the first, the administrator announces an allocation rule and pricing rule. Individuals then choose to enter or not and the units are randomly awarded in the second stage.

The allocation and pricing rules are assumed uniform: individuals may enter once and entrants share the same probability of being drawn $(\pi)$, and a fee is required to enter $(T)$ and/or a fee is required if drawn $(P)$. The expected price $(\mu)$ is then $\mu=$ $T+\pi P$, and equilibrium entry occurs such that $\pi \hat{v}=\mu$, where $\hat{v}$ is the valuation of the minimum-valued entrant. Given $Q^{*}, T$ and $P$, the equilibrium number of entrants is $Q(\hat{v})=F_{\hat{v}}^{+}(v) N$, where $F_{\hat{v}}^{+}(v)$ is the complement of the distribution function evaluated at $\hat{v}$.

The total value generated by the lottery is the sum of the fee revenues and the surplus value realized by those who receive units. Expected revenues are $\mu Q(\hat{v})=$ $T Q(\hat{v})+P Q^{*}$, so a unit change in $P$ maintains $\mu$ and $Q(\hat{v})$ constant if $T$ changes by $\pi$ units: $d T / d P=-\pi$. Revenue equivalence is established in the following proposition:

Proposition 1. Given $Q^{*}$ and $F(v)$, expected revenues are equal over uniform lotteries if and only if the number of entrants is equal.

Without loss of generality, if $T=0$ then $P=\hat{v}$ can be interpreted as the price of a unit and $Q(\hat{v})$ as the lottery demand function. Consumer surplus $(C S)$ conditional upon $Q^{*}$ and $\hat{v}$ is uniformly random over the $\left(\begin{array}{c}Q(\hat{v}) \\ Q^{*}\end{array}\right)$ sets of entrants that may be drawn and the conditional mean is:

$$
E\left(C S \mid \hat{v}, Q^{*}\right)=\sum_{j=1}^{\left(\begin{array}{c}
Q(\hat{v}) \\
Q^{*}
\end{array}\right)}\left(\begin{array}{c}
Q(\hat{v}) \\
Q^{*}
\end{array}\right)^{-1}\left(V(j)-\hat{v} Q^{*}\right)=Q^{*}\left(\frac{1}{Q(\hat{v})} \sum_{i=1}^{Q(\hat{v})} v(i)-\hat{v}\right)
$$

where $V(j)$ denotes the sum of the private values comprising the $j$ th combination of 
entrants. The second equality expresses expected surplus as a linear function of the deviation between the mean valuation conditional upon price-or average demand price (Mumy and Hanke, 1975)-and the price. The deviation is then both the mean surplus per-unit of supply and the marginal mean surplus of a unit. The conditional variance may also be expressed as a sum over sets of entrants or individual entrants:

$$
\begin{aligned}
\operatorname{Var}\left(C S \mid \hat{v}, Q^{*}\right)=\sum_{j=1}^{\left(\begin{array}{c}
Q(\hat{v}) \\
Q^{*}
\end{array}\right)}\left(\begin{array}{c}
Q(\hat{v}) \\
Q^{*}
\end{array}\right)^{-1}\left(C S(j)-E\left(C S \mid \hat{v}, Q^{*}\right)\right)^{2} \\
=\pi \sum_{i=1}^{Q(\hat{v})} v(i)^{2}-\left(\pi \sum_{i=1}^{Q(\hat{v})} v(i)\right)^{2}+2 \pi \frac{Q^{*}-1}{Q(\hat{v})-1} \sum_{i=1}^{Q(\hat{v})-1} v(i) \sum_{k=i}^{Q(\hat{v})} v(k+i)
\end{aligned}
$$

where $C S(j)$ denotes the consumer surplus of the $j$ th combination of entrants. Similar to revenues, (1) and (2) are independent of the pricing arrangement satisfying $\mu=T$ $+\pi P$. Proposition 2 establishes a sufficient condition for their equivalence:

Proposition 2. Given $Q^{*}$ and $F(v)$, the conditional mean and variance of consumer surplus are equal over uniform lotteries if the number of entrants is equal.

Propositions 1 and 2 imply that uniform lotteries are welfare equivalent: every combination of entry and user fees that attracts an equal number of entrants can be expected to yield the same revenue and consumer surplus and with equal variance. It follows that two-part pricing can not increase consumer welfare as in public sector settings such as those considered by Feldstein (1972) and Auerbach and Pellechio (1978), in which the quantity supplied is endogenous. However, the administrator has a variety of pricing options that yield a common set of outcomes.

Using continuous notation henceforth, the combinations of entry and user fees that maximize expected consumer surplus are determined from the solution to:

$$
\max _{\hat{v}} E\left(C S \mid Q^{*}\right)=Q^{*}\left(\frac{1}{Q(\hat{v})} \int_{0}^{Q(\hat{v})} v(k) d k-\hat{v}\right)
$$

Price can be seen to affect expected consumer surplus directly through its own effect 
and indirectly through the probability of being drawn and the total valuation, and expected consumer surplus is maximized where the marginal expected total value equals the marginal price. The first order condition for an interior maximum is:

$$
\frac{d E\left(C S \mid Q^{*}\right)}{d \hat{v}}=-\frac{Q^{\prime}(\hat{v})}{Q(\hat{v})^{2}} \int_{0}^{Q(\hat{v})} v(k) d k+Q^{\prime}(\hat{v}) \frac{\hat{v}}{Q(\hat{v})}-1=0
$$

The first two terms comprise the effect of price on the expected total value and the third term is the unit change in price. Noting the second term in absolute value is the price elasticity of demand, the solution may be expressed as a classic inverse elasticity pricing rule:

$$
\frac{\frac{1}{Q(\hat{v})} \int_{0}^{Q(\hat{v})} v(k) d k-\hat{v}}{\hat{v}}=\frac{1}{E_{Q, \hat{v}}}
$$

where $E_{Q, \hat{v}}$ is the price elasticity. (5) indicates that if an interior maximum to (3) exists, then it occurs where the inverse elasticity equals the percentage difference between the price and the average demand price (or the minimum and mean private valuations). Demand may be such that an interior solution does not exist (e.g., linear demand), and expected consumer surplus is maximized by setting price equal to zero. Alternatively, supply may be such that the optimal lottery approximates the competitive outcome. If an interior solution does exist then it can occur in the elastic or inelastic price range. ${ }^{4}$ Solving (4) or (5) for $\hat{v}$, the surplus maximizing price is:

$$
\hat{v}^{*}=\frac{1}{Q\left(\hat{v}^{*}\right)} \int_{0}^{Q\left(\hat{v}^{*}\right)} v(k) d k+\frac{Q\left(\hat{v}^{*}\right)}{Q^{\prime}\left(\hat{v}^{*}\right)}
$$

The price is equal to the difference between the conditional mean and the inverse

${ }^{4}$ From (4) it may be shown that at the optimum, $E_{Q, \hat{v}}>Q(\hat{v}) \hat{v} / \int_{0}^{Q(\hat{v})} v(k) d k<1$. 
hazard rate. ${ }^{5}$ From the equilibrium entry condition the optimal expected price is $\mu^{*}$ $=\pi^{*} \hat{v}^{*}$ and recalling $\mu=T+\pi P$, the combination of entry and user fees that maximizes expected consumer surplus satisfies $T=\pi^{*}\left(\hat{v}^{*}-P\right)$. The entry fee is then bound between zero and $\pi^{*} \hat{v}^{*}$ and the user fee between zero and $\hat{v}^{*}{ }^{6}$

Substituting (6) into (3), the maximum expected consumer surplus is:

$$
E\left(C S \mid \hat{v}^{*}, Q^{*}\right)=Q^{*} \frac{Q\left(\hat{v}^{*}\right)}{\left|Q^{\prime}\left(\hat{v}^{*}\right)\right|}
$$

Expressed in terms of the private value distribution, $E\left(C S \mid \hat{v}^{*}, Q^{*}\right)=Q^{*} F_{\hat{v}^{*}}^{+}(v) / f_{\hat{v}^{*}}(v)$, and it follows that an equivalent solution to the pricing problem is to establish price such that the conditional mean and inverse hazard rate of the distribution are equal.

A graphical example is depicted in Figure 1. Included are $Q^{*}$ and the inverse demand and average demand functions. Expected consumer surplus at any point is equal to the product of $Q^{*}$ and the vertical difference between the functions, and the optimal price is identified where the deviation is maximized. In addition to the maximum expected consumer surplus, the competitive surplus and the maximum consumer surplus given $\hat{v}^{*}$ are depicted in the diagram. ${ }^{7}$ Expected consumer surplus is about twice that of competitive pricing and about two-thirds that of efficient

$$
\begin{aligned}
& { }^{5} \text { In terms of } F(v),(5) \text { is }\left(\int_{\hat{v}}^{\bar{v}} v f(v) d v / F_{\hat{v}}^{+}(v)-\hat{v}\right) / \hat{v}=F_{\hat{v}}^{+}(v) / \hat{v} f_{\hat{v}}(v) \text { and }(6) \text { is } \\
& \hat{v}^{*}=\int_{\hat{v}}^{\bar{v}} v f(v) d v / F_{\hat{v}^{*}}^{+}(v)-F_{\hat{v}^{*}}^{+}(v) / f_{\hat{v}^{*}}(v) \text {. } \\
& 6
\end{aligned}
$$

The rule may be expressed in terms of any pricing arrangement satisfying $T=\pi(\hat{v}-$ $P$ ). For example, if $T=0$ then $P=\hat{v}$ so (5) is the pricing rule in user-pay lotteries. If instead $P=0$ then $T=\pi \hat{v}$ and $E_{Q, \hat{v}}=E_{Q, T} /\left(1-E_{Q, T}\right)$; to see this, note that $E_{Q, T}=$ $\left|T /\left[Q^{*} v^{\prime}(Q)-T\right]\right|$. Substituting $T=\pi \hat{v}$ into $E_{Q, \hat{v}}$ and rearranging yields $E_{Q, \hat{v}}=E_{Q, T}$ $/\left(1-E_{Q, T}\right)$. Substituting this into (5) yields the pricing rule in all-pay lotteries. 7

The latter results from what Davis and Kilian (2011) refer to as efficient rationing, whereby the $Q^{*}$ units are allocated to those individuals who would be willing to pay the clearing price $v\left(Q^{*}\right)$. 
rationing. The total value generated from competitive pricing relative to the optimal lottery can also be determined by comparing the conditional mean value given $v\left(Q^{*}\right)$ to that given $\hat{v}^{*}$. On average the lottery generates about twice the consumer surplus of competitive pricing but captures about half of the total value.

It is important to note the private value distribution in Figure 1 is sufficiently dense in the lower tail such that an interior maximum exists. As a counter example, if the values are uniformly distributed then the demand and average demand functions are linear, and expected consumer surplus is maximized by setting price equal to zero. Given the role the private value distribution serves in the optimization problem, the lottery pricing rule is investigated below across a rich class distributions.

\section{Numerical Analysis}

In this section the distributional conditions under which the optimal lottery price is positive (or zero) and the inverse elasticity rule are evaluated, and the performance of the lottery is compared to alternative pricing rules. The distribution of private values was specified as normal, Weibull, exponential, and beta, with each evaluated over a range of its respective parameter values. Reported here are results from the beta distribution. As noted by Taylor et. al (2003) and Koh et al. (2007), the beta distribution is appealing for analyses such as this because it has finite and scalable upper and lower bounds, and it nests common demand structures, including linear and convex demands, and demands containing concave and convex segments.

The beta density and cumulative distribution functions are:

$$
f(v ; a, b)=\frac{v^{a-1}(1-v)^{b-1}}{B(a, b)} \quad F(v ; a, b)=\int_{0}^{v} \frac{v^{a-1}(1-v)^{b-1}}{B(a, b)} d v
$$

where $v \in(0,1), a$ and $b$ are positive parameters, and $B(a, b)$ is the beta function. The mean and variance are $E(v)=a /(a+b)$ and $\operatorname{Var}(v)=a b /\left((a+b)^{2}(a+b+1)\right)$. The distribution is symmetric (uniform, mound-shaped, or bi-modal) if $a=b$, negatively skewed if $a<b$, and positively skewed if $a>b$ (see Gupta and Nadarajah (2004) for a comprehensive review). 
Given (8), the conditional mean and variance of consumer surplus are straightforward to derive (see Appendix A). The conditional mean is:

$$
E\left(C S \mid \hat{v}, Q^{*}\right)=Q^{*} \frac{\frac{a}{a+b} F_{\hat{v}}^{+}(v ; a+1, b)}{F_{\hat{v}}^{+}(v ; a, b)}-\hat{v} Q^{*}
$$

where $F_{\hat{v}}^{+}($.$) is the complement of F($.$) evaluated at \hat{v}$. The conditional variance is:

$\operatorname{Var}\left(C S \mid \hat{v}, Q^{*}\right)=Q^{*} \frac{a(a+1)}{(a+b)(a+b+1)} \frac{F_{\hat{v}}^{+}(v ; a+2, b)}{F_{\hat{v}}^{+}(v ; a, b)}-Q^{*}\left(\frac{a}{a+b} \frac{F_{\hat{v}}^{+}(v ; a+1, b)}{F_{\hat{v}}^{+}(v ; a, b)}\right)^{2}$

(9) and (10) are trivial to calculate with standard statistical software and both converge, respectively, upon $E(v)$ and $\operatorname{Var}(v)$ as $\hat{v} \rightarrow 0$ and $Q^{*} \rightarrow 1$.

\section{A. Inverse Elasticity Pricing and Optimal Entry}

The inverse elasticity pricing rule (5) with beta distributed values is:

$$
\frac{\frac{a}{a+b} \frac{F^{+}(v ; a+1, b)}{F^{+}(v ; a, b)}-v}{v}=\frac{F^{+}(v ; a, b)}{v f(v ; a, b)}
$$

The left term is the percentage difference between the price and average demand price and the right term is the inverse price elasticity (see Appendix B). Given $a$ and $b$, both terms are evaluated over the unit interval to determine the valuation $\hat{v}^{*} \geq 0$ satisfying (11) and the optimal fraction of entrants $F_{\hat{v}^{*}}^{+}(v ; a, b)$. The expected price $\mu^{*}$ is then determined from the equilibrium entry condition $\mu^{*}=\pi^{*} \hat{v}^{*}$ and the optimal entry and user fees $(T, P)$ from $T=\pi^{*}\left(\hat{v}^{*}-P\right) .^{8}$

8

$F_{\hat{v}^{*}}^{+}(v ; a, b)$ can not be expressed in closed form but can be modeled precisely in terms of $a$ and $b$ with the fractional dependent variable models proposed by Papke and Wooldridge (1996). Using the $n=72$ values from Table 1 as an example, more than $99 \%$ of the variation in $F_{\hat{v}^{*}}^{+}(v ; a, b)$ can be explained by first and second orders of $a$ and $b$ and an interaction term. The fractional logit estimation results are: 
The beta parameters were varied in 0.1 unit increments beginning from $a=$ 0.1 and $b=0.1$. The surplus maximizing price is positive for parameter values ( $a<$ $0.5, b \geq 0.1)$ and ( $0.5 \leq a \leq 0.7, b \geq 0.6)$. If $a \geq 0.8$, surplus is maximized if price is zero. The optimal price is then zero under linear demand $(a=b=1)$ and positive for power function distributions where $(a \leq 0.7, b=1)$. Figure 2 depicts inverse demand curves for $(a, b)$ pairs such that the optimal price excludes at least twenty-five percent of the population from entering: $F_{\hat{v}^{*}}^{+}(v ; a, b) \leq 0.75$. In all cases the curves are reasonable representations of consumer demand; they are either strictly convex at all prices or transition from concave to convex as price approaches zero.

The optimal fraction of entrants and the inverse elasticity solution are reported in Table 1 for $(a, b)$ pairs between $(0.1,0.1)$ and $(0.8,1.0){ }^{9}{ }^{9}$ The effectiveness of price in screening low-valued individuals can be seen in the first half of the table; given $a$, as $b \rightarrow 1$ the density of values increases in the lower tail of the distribution so the optimal price excludes an increasing fraction of the population. The shaded cells identify the thirty-two cases depicted in Figure 2 where at least onefourth of the population is excluded; in half of these less than half of the population enters, and in one fourth less than one third enters.

The second half of Table 1 describes the inverse elasticity solution (11). The reported values are the inverse price elasticity or the mean unit surplus relative to the price at the optimum. Demand in all cases is inelastic if the surplus maximizing price is positive.$^{10}$ Focusing upon the shaded cells, the results indicate that a 10 percent

$$
F_{\hat{v}^{*}}^{+}(v ; a, b)=G\left(-7.492 a+4.758 b+2.735 a b-2.377 a^{2}-2.851 b^{2}-0.178\right)
$$

where $G($.$) is the logistic function and the terms in parentheses are asymptotic z-$ scores in absolute value.

${ }^{9}$ Results for $a<0.8$ and $b>1$ are available in an appendix.

10

For comparison, the $(a, b)$ pairs yielding an interior solution at the unit elastic point (to at least three decimal places) are $(0.1,2.79),(0.2,3.42),(0.3,4.25),(0.4,5.40)$, $(0.5,7.03),(0.6,9.54),(0.7,13.76)$. The optimal fraction of entrants in these cases ranges from seven percent to thirty-one percent of the population. 
change in price from the optimum affects entry between 0.6 percent and 4.3 percent. Alternatively, the return to the average entrant if drawn is 2.5 to 10 times greater than the price. In the remainder of the section the performance of the optimal lottery is compared to alternative pricing rules.

\section{B. Comparison to Zero Pricing}

Table 2 reports the maximum expected consumer surplus relative to zero pricing. Attention is restricted to cases where the optimal price excludes at least twenty-five percent of the population (see Table 1). Results in the first half of Table 2 indicate that as much as 3.5 times more consumer surplus can be expected if the optimal price is established. ${ }^{11}$ And consistent with Table 1, the relative surplus of the lottery increases as the density of values increases in the lower tail of the distribution.

If supply is specified, the variance of the conditional distribution of consumer surplus (10) may be calculated, and together with the mean (9), the probability of realizing any particular surplus value under either pricing arrangement may be determined. Reported in parentheses in Table 2 are the probabilities that the optimal price (zero price) yields less (more) consumer surplus than the mean consumer surplus from the zero price (optimal price) for $Q^{*}=50$; formally: $\operatorname{Prob}\left(\left(C S \mid \mu=\mu^{*}\right)\right.$ $<E(C S \mid \mu=0))$ and $\operatorname{Prob}\left((C S \mid \mu=0)>E\left(C S \mid \mu=\mu^{*}\right)\right) .{ }^{12}$ In nearly all cases, the probabilities are less than 0.01 , indicating that $C S$ under the optimal price can be expected to exceed the mean $C S$ from zero pricing with near certainty. And with similar probability, $C S$ under zero pricing will be less than the mean $C S$ from optimal

11

However, the maximum expected consumer surplus is less than twice that of zero pricing in many of the cases, so a practical question to confront is whether moderate gains in consumer surplus justify excluding a potentially sizable fraction of the population from access to the lottery. As an entrant with valuation $\hat{v}^{*}$ derives zero expected value if $\mu=\mu^{*}$, the fraction of the population preferring $\mu^{*}$ is necessarily less than $F_{\hat{v}^{*}}^{+}(v ; a, b)$ reported in Table 1. Results in several cases indicate that if the price is chosen by referendum, $\mu^{*}$ will be majority-preferred over zero-pricing. 12

This quantity was selected because the distribution of the sum of $Q^{*}$ independent private valuations converges rapidly to the normal distribution for $Q^{*}>30$. 
pricing. The notable exception is $a=0.5$ and $b=1.0$, where the probability is about 0.15 that less (more) $C S$ is realized from optimal pricing (zero pricing) than is expected from zero pricing (optimal pricing).

\section{Comparison to Competitive Pricing}

Lottery performance is next compared to competitive pricing. As allocative inefficiency is the predominant criticism of lotteries, we begin by assessing the efficiency of competitive pricing relative to zero pricing and consumer surplus maximizing pricing. Relative efficiency is defined as the ratio of the total value of the competitive allocation to the expected total value of the respective lottery allocation. For consistency over distributions, supply is expressed relative to the optimal fraction of entrants. Two quantities are considered: $Q^{*}=0.10 F_{\hat{v}^{*}}^{+}(v)$ and $Q^{*}=0.25 F_{\hat{v}^{*}}^{+}(v)$ so entrants have, respectively, a one-in-ten and one-in-four chance of being drawn. ${ }^{13}$

As shown in Table 3, the efficiency criticism clearly has merit in the case of the zero price lottery, with competitive pricing generating between 3 and 10 times more value. In contrast, large efficiency gains are realized if price is established at the optimum, and the relative efficiency values are robust to the private value distribution, with competitive pricing capturing between 1.3 and 2.1 times more value. ${ }^{14}$ Relative consumer surplus is reported in Table 4 . For both the optimal and zero price lotteries the results indicate that expected consumer surplus can exceed that of competitive pricing by an order of magnitude or more. The difference diminishes as supply increases and as the density increases in the lower tail of the

13

Given $Q^{*}$, the shortage of units relative to the population may be inferred from Table 1. For example, if $a=b=0.1$ then about two-thirds enter, so if $Q^{*}=$ $0.10 F_{\hat{v}^{*}}^{+}(v)$ about six percent of the population receives units.

14

The efficiency of the optimal lottery relative to the zero price lottery may also be determined from Table 3; for each $(a, b)$ combination it is equal to the ratio of reported pair of relative efficiency values. For example, for $(a=0.1, b=1.0)$ and $Q^{*}$ $=0.10 F_{\hat{v}^{*}}^{+}(v)$, the optimal lottery captures on average $10.1 / 2.0 \approx 5.0$ times more than the zero price lottery if $Q^{*}=0.10 F_{\hat{v}^{*}}^{+}(v)$. 
distribution. In fact, in several cases the consumer surplus from competitive pricing is greater than the mean surplus from the zero-price lottery.

On the other hand, at least 4 times more surplus value can be expected from the optimal lottery for $Q^{*}=0.10 F_{\hat{v}^{*}}^{+}(v)$ and at least 2 times more for $Q^{*}=0.25 F_{\hat{v}^{*}}^{+}(v)$. Similar to the zero-price lottery, the probability that consumer surplus in the optimal lottery exceeds that from competitive pricing may be calculated. In all cases reported in Table 5 the results indicate that the optimal lottery will yield greater consumer surplus in more than ninety-nine percent of repeated samples.

In summary, the expected net benefits of the optimal lottery well exceed that of competitive pricing, while only moderate efficiency losses are incurred. Of course, the lottery also generates revenues otherwise foregone under zero pricing, though increasing price from the optimum or distributing a portion of the units competitively will further increase revenues. We turn to this issue in the next section.

\section{Hybrid Mechanisms}

Before concluding, the model is extended to the setting where a fraction of supply $(\alpha)$ is rationed by price and the remainder simultaneously by lottery. A similar hybrid mechanism was investigated by Evans et al. (2009), with a single unit of the good awarded through a zero-price lottery to individuals unsuccessful in a first-round auction. We characterize the pair of prices that maximizes the collective consumer surplus and numerically evaluate the performance of the mechanism against competitive pricing $(\alpha=1)$ and the base $(\alpha=0)$ lottery.

Entry in the lottery for $(1-\alpha) Q^{*}$ units satisfies the condition $\mu=\pi \hat{v}$, where $\mu$ again references the expected price $(\mu=T+\pi P)$ and the probability of being drawn is $\pi=(1-\alpha) Q^{*} /\left(Q(\hat{v})-\alpha Q^{*}\right)$. The clearing price $\widetilde{v}$ for the $\alpha Q^{*}$ remaining units satisfies the condition $v\left(\alpha Q^{*}\right)-\widetilde{v}=\pi v\left(\alpha Q^{*}\right)-\mu$, so a risk neutral individual with valuation $v\left(\alpha Q^{*}\right)$ is indifferent to purchasing a unit outright for $\widetilde{v}$ and entering the lottery. Expected revenues are $\alpha Q^{*} \widetilde{v}+T\left(Q(\hat{v})-\alpha Q^{*}\right)+(1-\alpha) Q^{*} P$, where $T$ and $P$ are the lottery entry and user fees. The surplus maximization problem is written: 


$$
\max _{\widetilde{v}, \hat{v}} E\left(C S \mid Q^{*}, \alpha\right)=\int_{0}^{\alpha Q^{*}} v(k) d k-\alpha Q^{*} \widetilde{v}+(1-\alpha) Q^{*}\left(\frac{1}{Q(\hat{v})-\alpha Q^{*}} \int_{\alpha Q^{*}}^{Q(\hat{v})} v(k) d k-\hat{v}\right)
$$

The first two terms represent the competitive consumer surplus and the third set of terms is the expected consumer surplus of the lottery. Solving the competitive clearing condition for $\widetilde{v}$ and substituting into (13), the problem reduces to a choice of $\hat{v}$. Entry in the lottery may be determined from the resulting first order condition and the optimal clearing price $\tilde{v}^{*}$ and lottery expected price $\mu^{*}$ from the respective equilibrium conditions. The solution is described by the pair of valuations:

$$
\hat{v}^{*}=\frac{1}{Q(\hat{v})} \int_{\alpha Q^{*}}^{Q(\hat{v})} v(k) d k+\frac{\alpha Q^{*} v\left(\alpha Q^{*}\right)}{Q(\hat{v})}+\frac{Q(\hat{v})-\alpha Q^{*}}{Q^{\prime}(\hat{v})} \quad \tilde{v}^{*}=\left(1-\pi^{*}\right) v\left(\alpha Q^{*}\right)+\pi^{*} \hat{v}^{*}
$$

As with the original problem, the lottery component of the solution can be expressed as a function of the inverse price elasticity, converging to (5) as $\alpha \rightarrow 0$.

Substituting (14) into (13), the maximum expected consumer surplus is:

$$
E\left(C S \mid \hat{v}^{*}, \widetilde{v}^{*}, \alpha, Q^{*}\right)=\int_{0}^{\alpha Q^{*}} v(k) d k-\alpha Q^{*} v\left(\alpha Q^{*}\right)+(1-\alpha) Q^{*} \frac{Q\left(\hat{v}^{*}\right)}{\left|Q^{\prime}\left(\hat{v}^{*}\right)\right|}
$$

The first two terms is the consumer surplus resulting if the $\alpha Q^{*}$ were sold at the clearing rate $v\left(\alpha Q^{*}\right)$ and the third term is the expected consumer surplus generated by the lottery. Just as the mechanism generates more revenues than the base lottery, expected consumer surplus diminishes, with the reduction in consumer surplus from the lottery less than offset by the consumer surplus resulting from competitively rationing a fraction of supply. ${ }^{15}$

For final comparison, expected consumer surplus and revenues are compared numerically to the base lottery and competitive pricing with beta distributed private

15

Alternatively, the hybrid optimization problem can be viewed as a constrained version of the earlier problem in which $\alpha=0$, and therefore the maximum expected consumer surplus is necessarily reduced. 
values. The first half of Table 5 reports consumer surplus for $\alpha=0.25$ and $\alpha=0.75$ relative to $\alpha=0$ given $Q^{*}=0.10 F_{\hat{v}^{*}}^{+}(v ; a, b) .{ }^{16}$ In both cases the results are robust to the private value distribution, with the mean surplus in the hybrid lottery consistently about eighty percent of the surplus generated by the lottery alone for $\alpha=0.25$ and between thirty and forty percent of the surplus for $\alpha=0.75$. The second half of Table 5 reports the revenues generated from competitive pricing relative to the base and hybrid lotteries. If the lottery is used exclusively, revenues are between one-sixth and one-twentieth of that from competitive pricing. However, revenues rise sharply if even a small portion of supply is sold outright. For $\alpha=0.25$ the relative revenues are between 2.5 and 3.7 greater than the hybrid lottery, and if $\alpha=0.75$ the competitive revenues are consistently about thirty-percent greater than the hybrid lottery.

\section{Conclusions}

The historic rationale for rationing certain classes of goods or assigning duties by lottery is based in fairness, in that entrants share a positive though not necessarily equal probability of being drawn. Public preferences and efficiency over competing mechanisms have also served to explain their use. The current paper demonstrated that lotteries can also be desirable on grounds of generating consumer surplus if price is appropriately established, and particularly so if awarded units are non-transferable. The solution to the lottery pricing problem was characterized by an inverse elasticity rule with the optimal price solely dependent upon the distribution of individual private valuations. Numerical analysis performed across a broad class of distributions indicated that the lottery can indeed yield sizable gains in consumer surplus over competitive pricing and zero pricing while maintaining a high degree of efficiency in the allocation.

In concluding, it is important to note that the optimal lottery is straightforward to implement if the distribution of private values is known.

\footnotetext{
${ }^{16}$ Results for $Q^{*}=0.25 F_{\hat{v}^{*}}^{+}(v ; a, b)$ are available in an appendix.
} 
According to the pricing rule developed here, the optimum is attained when price is established such that the conditional mean equals the inverse hazard rate of the distribution. If the distribution is unknown, then the mechanism must induce individuals to reveal their private valuations and might appear as a variant of the random-price auctions considered in Shogren et al. (2001), so that the optimal price being endogenously determined by bidders. Considering the increasing relatively scarcity of an array of public resource stocks and the on-going adoption of lotteries for allocating access rights, practical implementation and experimental analysis of optimal public lotteries are natural topics for future work. 


\section{References}

Auerbach, A. J. and A. J. Pellechio. 1978. The Two-Part Tariff and Voluntary Market Participation. Quarterly Journal of Economics, 92(4): 571-587.

Boyce, J. R. 1994. Allocation of Goods by Lottery. Economic Inquiry, 32(3): 457-76.

Bulow, J. and P. Klemperer. 2012. Regulated Prices, Rent Seeking, and Consumer Surplus. Journal of Political Economy, 120(1): 160-186.

Buschena, D. E., T. L. Anderson, and J. L. Leonard. 2001. Valuing Non-Marketed Goods: The Case of Elk Permit Lotteries. Journal of Environmental Economics and Management, 4(1): 33-43.

Bush, G. W., September 15, 2005, Rebuilding After Hurricane Katrina. Presidential speech to the nation presented from New Orleans, Louisiana.

Che, Y. K., I. Gale, and J. Kim. 2012. Assigning Resources to Budget-Constrained Agents, forthcoming in Review of Economic Studies.

Condorelli, D., 2012. What Money Can't Buy: Efficient Mechanism Design with Costly Signals. Games and Economic Behavior, 75(2): 613-624.

Davis, L. W. and L. Kilian. 2011. The Allocative Cost of Price Ceilings in the U.S. Residential Market for Natural Gas. Journal of Political Economy, 119(2): 212-241.

Evans, M. F., C. A. Vossler and N. E. Flores. 2009. Hybrid Allocation Mechanisms for Publicly Provided Goods. Journal of Public Economics, 93(1-2): 311325.

Feldstein, M. 1972. Equity and Efficiency in Public Sector Pricing: The Optimal Two-Part Tariff. Quarterly Journal of Economics, 86(2): 175-187.

Glaeser, E. L. and Erzo F. P. Luttmer. 2003. The Misallocation of Housing Under Rent Control. American Economic Review, 93(4): 1027-1046.

Gupta, K. and S. Nadarajah eds. 2004. Handbook of Beta Distribution and Its Applications. New York, NY: Marcel Dekker, Inc.

Kahneman, D., J. L. Knetsch, and R. H. Thaler. 1986. Fairness and the Assumptions of Economics. Journal of Business, 59(4): S285-S300.

Koh, W. T. H., Z. Yang and L. Zhu. 2007. Lottery Rather than Waiting-Line Auction. Social Choice and Welfare, 27(2): 289-310. 
Mumy, G. E. and S. H. Hanke. 1975. Public Investment Criteria for Underpriced Public Products. American Economic Review, 65(4): 712-720.

National Park Service. 2006. Colorado River Management Plan. Grand Canyon National Park, Office of Planning and Compliance, U.S. Department of Interior, 1-42.

Papke, L. E. and J. M. Wooldridge. 1996. Econometric Methods for Fractional Response Variables with an Application to 401(K) Plan Participation Rates. Journal of Applied Econometrics, 11(6): 619-632.

Scrogin, D. 2005. Lottery-Rationed Public Access Under Alternative Tariff Arrangements: Changes in Quality, Quantity, and Expected Utility. Journal of Environmental Economics and Management, 50(1): 189-211.

Shogren, J. F., M. Margolis, C. Koo, and J. A. List. 2001. A Random nth-Price Auction. Journal of Economic Behavior and Organization, 46(4): 409-421.

Taylor, G. A., K. K. Tsui and L. Zhu. 2003. Lottery or Waiting-line Auction? Journal of Public Economics, 87(5-6): 1313-1334.

Tobin, J. 1970. On Limiting the Domain of Inequality. Journal of Law and Economics, 13(2): 263-277. 
Table 1. The Uniform Lottery with Beta Distributed Private Values: $E(C S)$ Maximizing Entry and the Inverse Price Elasticity Solution

\begin{tabular}{|c|c|c|c|c|c|c|c|c|c|c|c|c|c|c|c|c|c|c|c|c|}
\hline \multirow[b]{2}{*}{$(a, b)$} & \multicolumn{10}{|c|}{$F_{\hat{v}^{*}}^{+}(v ; a, b) \times 100$} & \multicolumn{10}{|c|}{$\begin{array}{l}\text { The Inverse Price Elasticity of Demand }\left(1 / E_{Q, v}\right) \\
\qquad \text { or } \\
\text { The Mean Unit Surplus Relative to the Price }\end{array}$} \\
\hline & 0.1 & 0.2 & 0.3 & 0.4 & 0.5 & 0.6 & 0.7 & 0.8 & 0.9 & 1.0 & 0.1 & 0.2 & 0.3 & 0.4 & 0.5 & 0.6 & 0.7 & 0.8 & 0.9 & 1.0 \\
\hline 0.1 & 63 & 47 & 38 & 32 & 28 & 25 & 23 & 21 & 19 & 17 & 16.0 & 8.3 & 5.8 & 4.5 & 3.7 & 3.2 & 2.8 & 2.6 & 2.3 & 2.1 \\
\hline 0.2 & 83 & 70 & 61 & 55 & 49 & 45 & 41 & 39 & 36 & 34 & 23.0 & 11.2 & 7.5 & 5.8 & 4.6 & 3.9 & 3.5 & 3.1 & 2.8 & 2.5 \\
\hline 0.3 & 92 & 83 & 76 & 70 & 65 & 61 & 58 & 54 & 51 & 49 & 36.2 & 16.2 & 10.4 & 7.6 & 6.2 & 5.1 & 4.5 & 3.9 & 3.5 & 3.2 \\
\hline 0.4 & 96 & 91 & 86 & 82 & 78 & 74 & 71 & 70 & 65 & 63 & 65.5 & 25.7 & 15.5 & 11.1 & 8.7 & 7.1 & 6.1 & 5.2 & 4.7 & 4.2 \\
\hline 0.5 & 100 & 96 & 93 & 90 & 87 & 85 & 82 & 79 & 77 & 75 & -- & 49.0 & 27.0 & 18.2 & 13.6 & 10.9 & 9.0 & 7.8 & 6.8 & 6.0 \\
\hline 0.6 & -- & 100 & 97 & 96 & 94 & 92 & 90 & 89 & 87 & 85 & -- & -- & 59.8 & 37.3 & 25.0 & 19.8 & 15.5 & 13.0 & 11.1 & 9.6 \\
\hline 0.7 & -- & -- & 100 & 100 & 100 & 97 & 96 & 95 & 94 & 93 & -- & -- & -- & -- & -- & 48.4 & 35.6 & 29.1 & 23.8 & 19.5 \\
\hline 0.8 & -- & -- & -- & -- & -- & 100 & 100 & 100 & 100 & 100 & -- & -- & -- & -- & -- & -- & -- & -- & -- & -- \\
\hline
\end{tabular}

Note: The shaded cells identify the cases where the optimal price excludes at least twenty-five percent of the population. 
Table 2. Relative Expected Consumer Surplus:

E(CS) Maximizing Pricing Versus Zero Pricing

Relative Expected Consumer Surplus:

$E(C S)$ Maximizing Pricing Versus Zero Pricing

\begin{tabular}{|c|c|c|c|c|c|c|c|c|c|c|}
\hline$(a, b)$ & 0.1 & 0.2 & 0.3 & 0.4 & 0.5 & 0.6 & 0.7 & 0.8 & 0.9 & 1.0 \\
\hline 0.1 & $\begin{array}{c}1.5 \\
(0.00 \\
0.00)\end{array}$ & $\begin{array}{c}1.9 \\
(0.00 \\
0.00)\end{array}$ & $\begin{array}{c}2.2 \\
(0.00 \\
0.00)\end{array}$ & $\begin{array}{c}2.4 \\
(0.00 \\
0.00)\end{array}$ & $\begin{array}{c}2.7 \\
(0.00 \\
0.00)\end{array}$ & $\begin{array}{c}2.9 \\
(0.00 \\
0.00)\end{array}$ & $\begin{array}{c}3.0 \\
(0.00 \\
0.00)\end{array}$ & $\begin{array}{c}3.2 \\
(0.00 \\
0.00)\end{array}$ & $\begin{array}{c}3.3 \\
(0.00 \\
0.00)\end{array}$ & $\begin{array}{c}3.4 \\
(0.00 \\
0.00)\end{array}$ \\
\hline 0.2 & -- & $\begin{array}{c}1.3 \\
(0.01 \\
0.00)\end{array}$ & $\begin{array}{c}1.4 \\
(0.00 \\
0.00)\end{array}$ & $\begin{array}{c}1.5 \\
(0.00 \\
0.00)\end{array}$ & $\begin{array}{c}1.6 \\
(0.00 \\
0.00)\end{array}$ & $\begin{array}{c}1.7 \\
(0.00 \\
0.00)\end{array}$ & $\begin{array}{c}1.8 \\
(0.00 \\
0.00)\end{array}$ & $\begin{array}{c}1.8 \\
(0.00 \\
0.00)\end{array}$ & $\begin{array}{c}1.9 \\
(0.00 \\
0.00)\end{array}$ & $\begin{array}{c}1.9 \\
(0.00 \\
0.00)\end{array}$ \\
\hline 0.3 & -- & -- & -- & $\begin{array}{c}1.2 \\
(0.03 \\
0.01)\end{array}$ & $\begin{array}{c}1.3 \\
(0.02 \\
0.01)\end{array}$ & $\begin{array}{c}1.3 \\
(0.01 \\
0.00)\end{array}$ & $\begin{array}{c}1.4 \\
(0.01 \\
0.00)\end{array}$ & $\begin{array}{c}1.4 \\
(0.00 \\
0.00)\end{array}$ & $\begin{array}{c}1.4 \\
(0.00 \\
0.00)\end{array}$ & $\begin{array}{c}1.5 \\
(0.00 \\
0.00)\end{array}$ \\
\hline 0.4 & -- & -- & -- & -- & -- & $\begin{array}{c}1.2 \\
(0.09 \\
0.06)\end{array}$ & $\begin{array}{c}1.2 \\
(0.07 \\
0.05)\end{array}$ & $\begin{array}{c}1.2 \\
(0.06 \\
0.04)\end{array}$ & $\begin{array}{c}1.2 \\
(0.05 \\
0.04)\end{array}$ & $\begin{array}{c}1.2 \\
(0.04 \\
0.03)\end{array}$ \\
\hline 0.5 & -- & -- & -- & -- & -- & -- & -- & -- & -- & $\begin{array}{c}1.1 \\
(0.16 \\
0.14)\end{array}$ \\
\hline
\end{tabular}

Note: In parentheses are $\operatorname{Prob}\left(\left(C S \mid \mu=\mu^{*}\right)<E(C S \mid \mu=0)\right)$ and $\operatorname{Prob}((C S \mid \mu=0)$ $\left.>E\left(C S \mid \mu=\mu^{*}\right)\right)$ for supply of $Q^{*}=50$. 
Table 3. Relative Efficiency:

Competitive Pricing Versus $E(C S)$ Maximizing Pricing and Zero Pricing

\begin{tabular}{|c|c|c|c|c|c|c|c|c|c|c|c|c|c|c|c|c|c|c|c|c|c|}
\hline \multirow[b]{2}{*}{$(a, b)$} & & \multicolumn{10}{|c|}{ 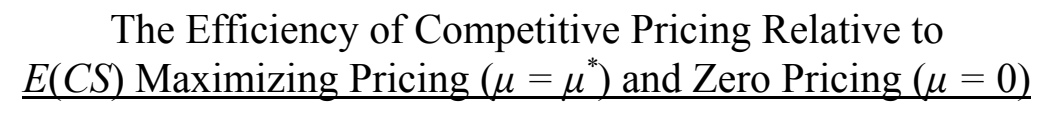 } & \multicolumn{10}{|c|}{ 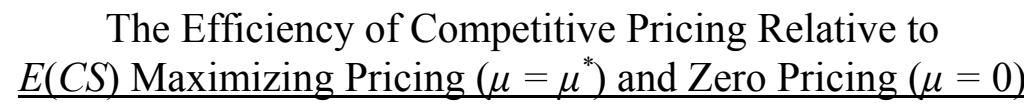 } \\
\hline & $\mu$ & 0.1 & 0.2 & 0.3 & 0.4 & 0.5 & 0.6 & 0.7 & 0.8 & 0.9 & 1.0 & 0.1 & 0.2 & 0.3 & 0.4 & 0.5 & 0.6 & 0.7 & 0.8 & 0.9 & 1.0 \\
\hline \multirow[t]{2}{*}{0.1} & $\mu=0$ & 2.9 & 3.0 & 4.0 & 5.0 & 6.0 & 6.9 & 7.7 & 8.6 & 9.4 & 10.1 & 2.0 & 3.0 & 4.0 & 4.9 & 5.7 & 6.5 & 7.1 & 7.8 & 8.3 & 8.9 \\
\hline & $\mu=\mu^{*}$ & 1.3 & 1.4 & 1.6 & 1.7 & 1.8 & 1.8 & 1.9 & 1.9 & 2.0 & 2.0 & 1.3 & 1.4 & 1.5 & 1.6 & 1.7 & 1.7 & 1.7 & 1.8 & 1.8 & 1.8 \\
\hline \multirow[t]{2}{*}{0.2} & $\mu=0$ & -- & 2.0 & 2.5 & 3.0 & 3.5 & 3.9 & 4.4 & 4.8 & 5.2 & 5.5 & \multirow[t]{2}{*}{-- } & 2.0 & 2.5 & 2.9 & 3.3 & 3.7 & 4.0 & 4.3 & 4.6 & 4.9 \\
\hline & $\mu=\mu^{*}$ & & 1.4 & 1.5 & 1.7 & 1.8 & 1.8 & 1.9 & 2.0 & 2.0 & 2.0 & & 1.4 & 1.5 & 1.6 & 1.7 & 1.7 & 1.8 & 1.8 & 1.8 & 1.8 \\
\hline \multirow[t]{2}{*}{0.3} & $\mu=0$ & -- & -- & -- & 2.3 & 2.6 & 3.0 & 3.2 & 3.5 & 3.8 & 4.0 & \multirow[t]{2}{*}{-- } & \multirow[t]{2}{*}{--} & \multirow[t]{2}{*}{-- } & 2.3 & 2.5 & 2.8 & 3.0 & 3.2 & 3.4 & 3.5 \\
\hline & $\mu=\mu^{*}$ & & & & 1.7 & 1.8 & 1.9 & 1.9 & 2.0 & 2.0 & 2.0 & & & & 1.6 & 1.7 & 1.7 & 1.8 & 1.8 & 1.8 & 1.8 \\
\hline \multirow[t]{2}{*}{0.4} & $\mu=0$ & -- & -- & -- & -- & -- & 2.5 & 2.7 & 2.9 & 3.1 & 3.2 & \multirow[t]{2}{*}{-- } & \multirow[t]{2}{*}{-- } & \multirow[t]{2}{*}{-- } & \multirow[t]{2}{*}{-- } & \multirow[t]{2}{*}{-- } & 2.3 & 2.5 & 2.6 & 2.7 & 2.9 \\
\hline & $\mu=\mu^{*}$ & & & & & & 1.9 & 1.9 & 2.0 & 2.1 & 2.1 & & & & & & 1.8 & 1.8 & 1.8 & 1.8 & 1.9 \\
\hline \multirow[t]{2}{*}{0.5} & $\mu=0$ & -- & -- & -- & -- & -- & -- & -- & -- & -- & 2.8 & -- & -- & -- & -- & -- & -- & -- & -- & -- & 2.5 \\
\hline & $\mu=\mu^{*}$ & & & & & & & & & & 2.1 & & & & & & & & & & 1.9 \\
\hline
\end{tabular}


Table 4. Relative Expected Consumer Surplus:

\section{$E(C S)$ Maximizing Pricing and Zero Pricing Versus Competitive Pricing}

\begin{tabular}{|c|c|c|c|c|c|c|c|c|c|c|c|c|c|c|c|c|c|c|c|c|c|}
\hline \multirow[b]{2}{*}{$(a, b)$} & \multirow[b]{2}{*}{$\mu$} & \multicolumn{10}{|c|}{$\begin{array}{c}E(C S) \text { from Optimal Pricing }\left(\mu=\mu^{*}\right) \text { and Zero Pricing }(\mu=0) \\
\text { Relative to Competitive Pricing }\end{array}$} & \multicolumn{10}{|c|}{$\begin{array}{c}E(C S) \text { from Optimal Pricing }\left(\mu=\mu^{*}\right) \text { and Zero Pricing }(\mu=0) \\
\text { Relative to Competitive Pricing }\end{array}$} \\
\hline & & 0.1 & 0.2 & 0.3 & 0.4 & 0.5 & 0.6 & 0.7 & 0.8 & 0.9 & 1.0 & 0.1 & 0.2 & 0.3 & 0.4 & 0.5 & 0.6 & 0.7 & 0.8 & 0.9 & 1.0 \\
\hline \multirow[t]{2}{*}{0.1} & $\mu=0$ & $>500$ & $>500$ & 197.5 & 30.5 & 10.0 & 4.8 & 2.9 & 2.0 & 1.5 & 1.2 & $>500$ & 86.5 & 9.6 & 3.3 & 1.8 & 1.2 & 0.9 & 0.7 & 0.6 & 0.5 \\
\hline & $\mu=\mu^{*}$ & $>500$ & $>500$ & 433.4 & 74.7 & 26.6 & 13.8 & 8.6 & 6.2 & 4.8 & 4.0 & $>500$ & 163.2 & 21.0 & 8.0 & 4.7 & 3.4 & 2.7 & 2.3 & 2.0 & 2.0 \\
\hline \multirow[t]{2}{*}{0.2} & $\mu=0$ & -- & $>500$ & 349.6 & 54.5 & 18.4 & 8.9 & 5.3 & 3.6 & 2.7 & 2.2 & -- & 148.5 & 16.8 & 5.8 & 3.2 & 2.1 & 1.6 & 1.3 & 1.1 & 1.0 \\
\hline & $\mu=\mu^{*}$ & -- & $>500$ & 498.8 & 83.3 & 29.8 & 15.1 & 9.4 & 6.7 & 5.1 & 4.2 & -- & 193.8 & 23.9 & 8.9 & 5.1 & 3.6 & 2.9 & 2.4 & 2.1 & 2.0 \\
\hline \multirow[t]{2}{*}{0.3} & $\mu=0$ & -- & -- & -- & 77.0 & 25.5 & 12.4 & 7.5 & 5.1 & 3.9 & 3.1 & -- & -- & -- & 8.1 & 4.4 & 2.9 & 2.2 & 1.8 & 1.6 & 1.4 \\
\hline & $\mu=\mu^{*}$ & -- & -- & -- & 95.6 & 32.9 & 16.6 & 10.3 & 7.2 & 5.6 & 4.5 & -- & -- & -- & 10.1 & 5.7 & 3.9 & 3.1 & 2.6 & 2.3 & 2.1 \\
\hline \multirow[t]{2}{*}{0.4} & $\mu=0$ & -- & -- & -- & -- & -- & 15.9 & 9.5 & 6.5 & 4.9 & 3.9 & -- & -- & -- & -- & -- & 3.7 & 2.8 & 2.3 & 1.9 & 1.7 \\
\hline & $\mu=\mu^{*}$ & -- & -- & -- & -- & -- & 18.5 & 11.3 & 7.9 & 6.0 & 4.9 & -- & -- & -- & -- & -- & 4.3 & 3.3 & 2.7 & 2.4 & 2.1 \\
\hline \multirow[t]{2}{*}{0.5} & $\mu=0$ & -- & -- & -- & -- & -- & -- & -- & -- & -- & 4.7 & -- & -- & -- & -- & -- & -- & -- & -- & -- & 2.0 \\
\hline & $\mu=\mu^{*}$ & -- & -- & -- & -- & -- & -- & -- & -- & -- & 5.3 & -- & -- & -- & -- & -- & -- & -- & -- & -- & 2.3 \\
\hline
\end{tabular}


Table 5. Relative Expected Consumer Surplus and Revenues in the Hybrid Lottery

Supply: $Q^{*}=0.10 F_{\hat{v}^{*}}^{+}(v ; a, b)$

\begin{tabular}{|c|c|c|c|c|c|c|c|c|c|c|c|c|c|c|c|c|c|c|c|c|c|}
\hline \multirow[b]{2}{*}{$(a, b)$} & \multirow[b]{2}{*}{$\begin{array}{c}\text { Fraction of } Q^{*} \\
\text { Sold Outright }(\alpha)\end{array}$} & \multicolumn{10}{|c|}{$E(C S)$ in the Hybrid Lottery Relative to the Base Lottery } & \multicolumn{10}{|c|}{$\begin{array}{l}\text { Expected Revenues from Competitive Pricing } \\
\text { Relative to the Hybrid Lottery }\end{array}$} \\
\hline & & 0.1 & 0.2 & 0.3 & 0.4 & 0.5 & 0.6 & 0.7 & 0.8 & 0.9 & 1.0 & 0.1 & 0.2 & 0.3 & 0.4 & 0.5 & 0.6 & 0.7 & 0.8 & 0.9 & 1.0 \\
\hline \multirow[t]{3}{*}{0.1} & 0 & 1 & 1 & 1 & 1 & 1 & 1 & 1 & 1 & 1 & 1 & 21.3 & 13.1 & 10.5 & 9.1 & 8.2 & 7.5 & 6.9 & 6.6 & 6.1 & 5.7 \\
\hline & 0.25 & 0.8 & 0.8 & 0.8 & 0.8 & 0.8 & 0.8 & 0.8 & 0.8 & 0.8 & 0.8 & 3.7 & 3.4 & 3.2 & 3.1 & 2.9 & 2.9 & 2.8 & 2.7 & 2.5 & 2.5 \\
\hline & 0.75 & 0.3 & 0.3 & 0.3 & 0.3 & 0.3 & 0.3 & 0.3 & 0.4 & 0.4 & 0.4 & 1.3 & 1.3 & 1.3 & 1.3 & 1.3 & 1.3 & 1.3 & 1.2 & 1.2 & 1.2 \\
\hline \multirow[t]{3}{*}{0.2} & 0 & -- & 1 & 1 & 1 & 1 & 1 & 1 & 1 & 1 & 1 & -- & 17.1 & 13.2 & 11.2 & 9.8 & 8.9 & 8.1 & 7.6 & 7.0 & 6.6 \\
\hline & 0.25 & -- & 0.8 & 0.8 & 0.8 & 0.8 & 0.8 & 0.8 & 0.8 & 0.8 & 0.8 & -- & 3.6 & 3.5 & 3.2 & 3.0 & 3.0 & 2.9 & 2.8 & 2.8 & 2.6 \\
\hline & 0.75 & -- & 0.3 & 0.3 & 0.3 & 0.3 & 0.3 & 0.3 & 0.4 & 0.4 & 0.4 & -- & 1.3 & 1.3 & 1.3 & 1.3 & 1.3 & 1.3 & 1.3 & 1.2 & 1.2 \\
\hline \multirow[t]{3}{*}{0.3} & 0 & -- & -- & -- & 1 & 1 & 1 & 1 & 1 & 1 & 1 & -- & -- & -- & 14.2 & 12.4 & 11.1 & 10.1 & 9.3 & 8.5 & 7.9 \\
\hline & 0.25 & -- & -- & -- & 0.8 & 0.8 & 0.8 & 0.8 & 0.8 & 0.8 & 0.8 & -- & -- & -- & 3.5 & 3.4 & 3.2 & 3.1 & 2.9 & 2.8 & 2.7 \\
\hline & 0.75 & -- & -- & -- & 0.3 & 0.3 & 0.3 & 0.3 & 0.3 & 0.4 & 0.4 & -- & -- & -- & 1.3 & 1.3 & 1.3 & 1.3 & 1.3 & 1.3 & 1.3 \\
\hline \multirow[t]{3}{*}{0.4} & 0 & -- & -- & -- & -- & -- & 1 & 1 & 1 & 1 & 1 & -- & -- & -- & -- & -- & 14.7 & 13.1 & 11.8 & 11.0 & 10.0 \\
\hline & 0.25 & -- & -- & -- & -- & -- & 0.8 & 0.8 & 0.8 & 0.8 & 0.8 & -- & -- & -- & -- & -- & 3.4 & 3.3 & 3.2 & 3.0 & 2.9 \\
\hline & 0.75 & -- & -- & -- & -- & -- & 0.3 & 0.3 & 0.3 & 0.4 & 0.4 & -- & -- & -- & -- & -- & 1.3 & 1.3 & 1.3 & 1.3 & 1.3 \\
\hline \multirow[t]{3}{*}{0.5} & 0 & -- & -- & -- & -- & -- & -- & -- & -- & -- & 1 & -- & -- & -- & -- & -- & -- & -- & -- & -- & 13.6 \\
\hline & 0.25 & -- & -- & -- & -- & -- & -- & -- & -- & -- & 0.8 & -- & -- & -- & -- & -- & -- & -- & -- & -- & 3.1 \\
\hline & 0.75 & -- & -- & -- & -- & -- & -- & -- & -- & -- & 0.4 & -- & -- & -- & -- & -- & -- & -- & -- & -- & 1.3 \\
\hline
\end{tabular}


Figure 1. An Expected Consumer Surplus Maximizing Lottery

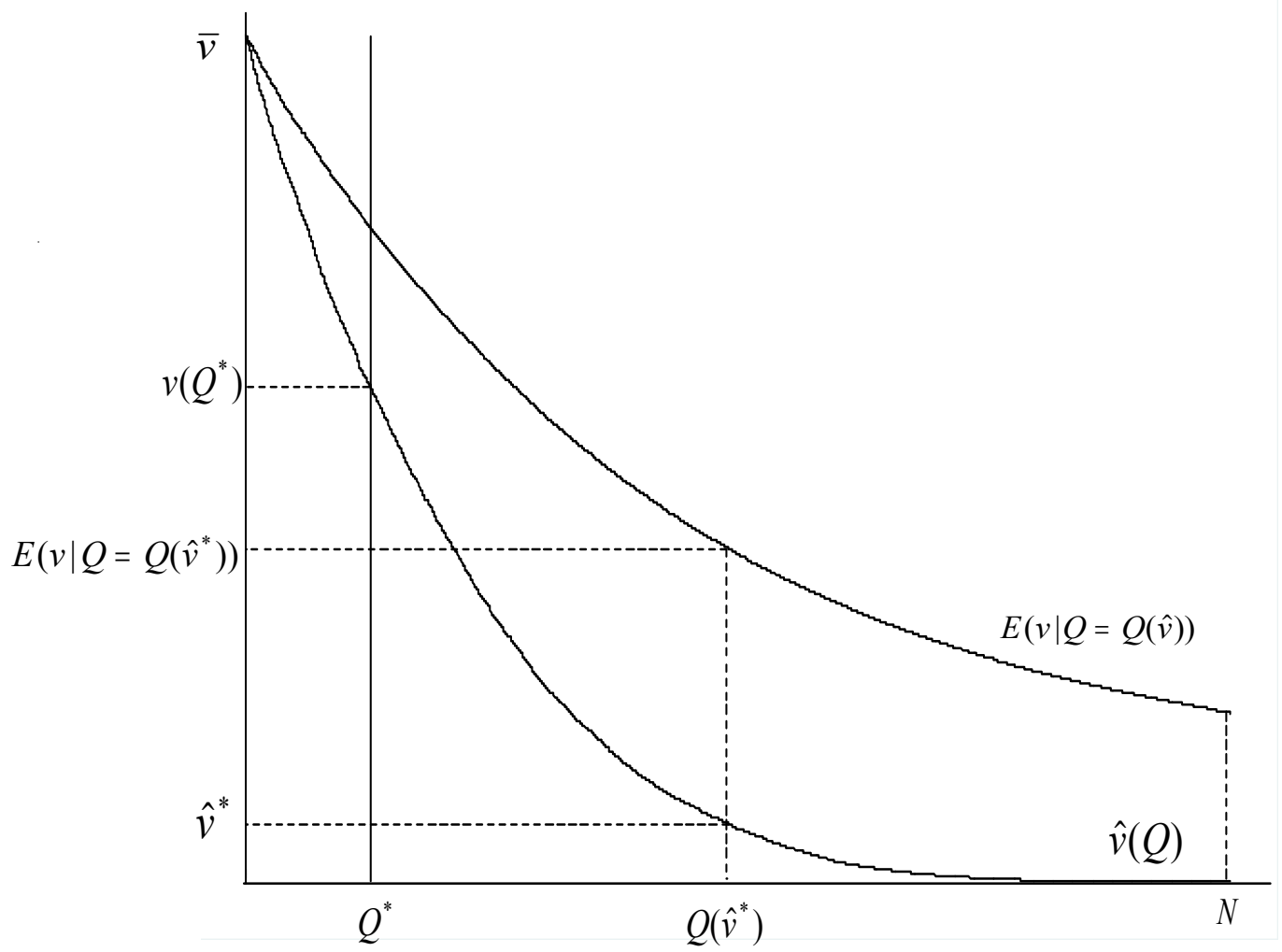

Note: The lower curve is the inverse demand curve and the upper curve is the inverse average demand curve or the mean private valuation conditional upon price. 
Figure 2. Selected Inverse Demand Curves

Under Beta Distributed Private Values

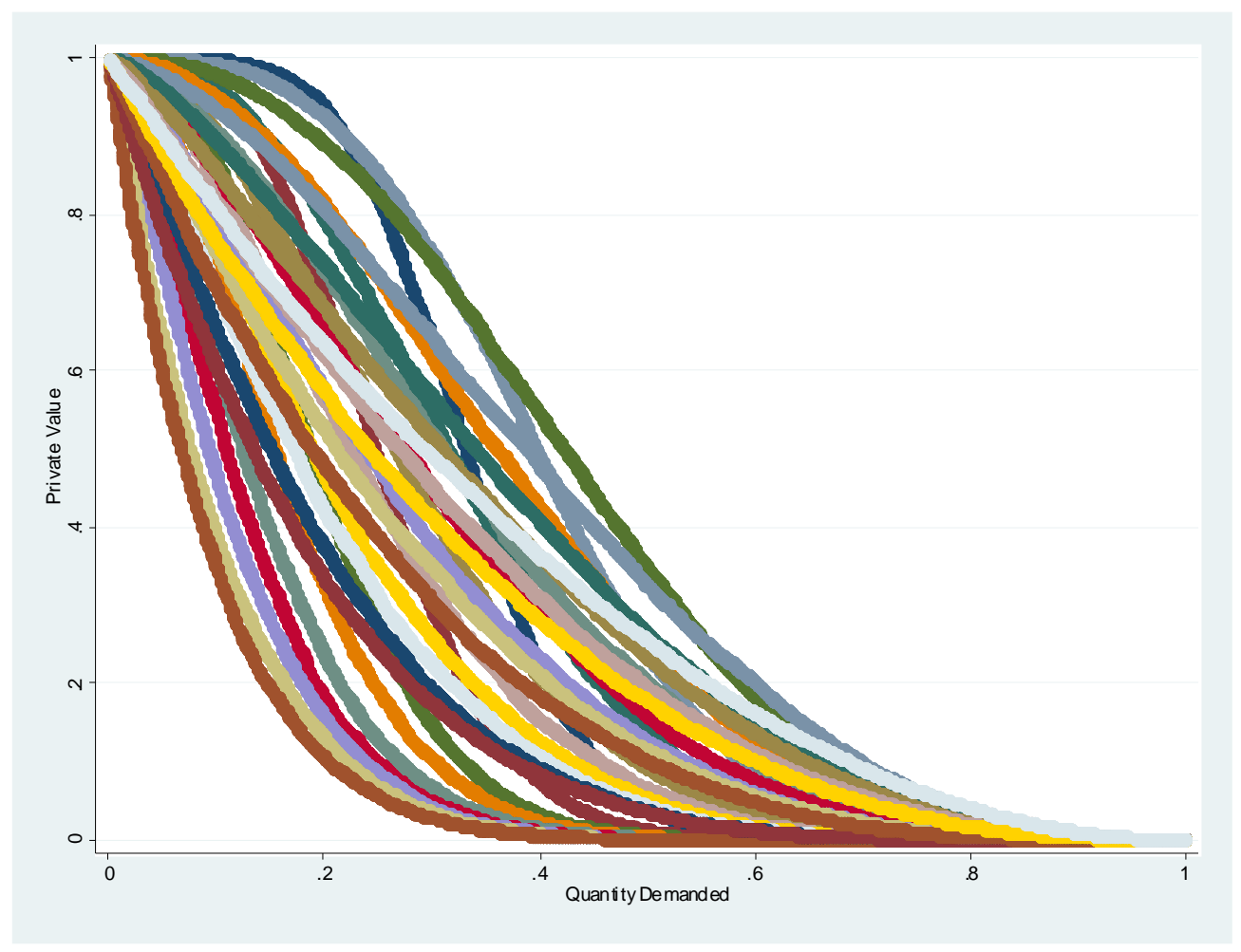

Note: The inverse demand curves are those such that the expected surplus maximizing price screens at least twenty-five percent of the population. The respective beta distributions are identified by the shaded cells in Table 1. 


\section{Appendix A. Derivation of the Conditional Mean and Variance of Consumer Surplus with Beta-Distributed Private Values}

Let the population of private values be distributed over the unit interval according to the distribution function $F(v)$ and density function $f(v)$. Expected consumer surplus given supply $Q^{*}$ and price $\hat{v}$ is:

$$
E\left(C S \mid \hat{v}, Q^{*}\right)=Q^{*} \frac{\int_{\hat{v}}^{1}(v-\hat{v}) f(v) d v}{F_{\hat{v}}^{+}(v)}
$$

where $F_{\hat{v}}^{+}(v)$ is the complement of $F(v)$ evaluated at $\hat{v}$. (A1) may be rewritten:

$$
E\left(C S \mid \hat{v}, Q^{*}\right)=Q^{*} \frac{\int_{\hat{v}}^{1} v f(v) d v}{F_{\hat{v}}^{+}(v)}-Q^{*} \hat{v}
$$

Substituting the beta distribution and density functions, conditional expected consumer surplus is:

$$
E\left(C S \mid \hat{v}, Q^{*}\right)=Q^{*} \frac{\frac{a}{a+b} F_{\hat{v}}^{+}(v ; a+1, b)}{F_{\hat{v}}^{+}(v ; a, b)}-Q^{*} \hat{v}
$$

To derive the conditional variance, the relation $\operatorname{Var}\left(C S \mid \hat{v}, Q^{*}\right)=E\left(C S^{2} \mid \hat{v}, Q^{*}\right)-\left(E\left(C S \mid \hat{v}, Q^{*}\right)\right)^{2}$ is employed. The conditional variance may be written:

$$
\operatorname{Var}(C S \mid \hat{v})=Q^{*} \frac{\int_{\hat{v}}^{1}(v-\hat{v})^{2} f(v) d v}{F_{\hat{v}}^{+}(v)}-Q^{*}\left(\frac{\int_{\hat{v}}^{1}(v-\hat{v}) f(v) d v}{F_{\hat{v}}^{+}(v)}\right)^{2}
$$

Substituting the beta distribution and density functions and rearranging, the conditional variance is:

$$
\operatorname{Var}\left(C S \mid \hat{v}, Q^{*}\right)=Q^{*} \frac{a(a+1)}{(a+b)(a+b+1)} \frac{F_{\hat{v}}^{+}(v ; a+2, b)}{F_{\hat{v}}^{+}(v ; a, b)}-Q^{*}\left(\frac{a}{a+b} \frac{F_{\hat{v}}^{+}(v ; a+1, b)}{F_{\hat{v}}^{+}(v ; a, b)}\right)^{2}
$$

As $\hat{v} \rightarrow 0$ and $Q^{*} \rightarrow 1$, (A3) and (A5) converge upon the mean and variance of the beta distribution. 


\section{Appendix B. Derivation of the Inverse Elasticity Pricing Rule (11) with Beta-Distributed Private Values}

From Appendix A, expected consumer surplus conditional upon price $\hat{v}$ and supply $Q^{*}$ is:

$$
E\left(C S \mid \hat{v}, Q^{*}\right)=Q^{*} \frac{\frac{a}{a+b} F_{\hat{v}}^{+}(v ; a+1, b)}{F_{\hat{v}}^{+}(v ; a, b)}-Q^{*} \hat{v}
$$

Given $Q^{*}$ the first order necessary condition for an interior maximum is:

$$
\frac{d E(C S \mid \hat{v})}{d \hat{v}}=\frac{a}{a+b} \frac{f(v ; a, b)}{F^{+}(v ; a, b)^{2}}-\frac{a}{a+b} \frac{f(v ; a+1, b)}{F^{+}(v ; a, b)}-\frac{a}{a+b} \frac{f(v ; a, b) F(v ; a+1, b)}{F^{+}(v ; a, b)^{2}}-1=0
$$

Rearranging yields:

$$
\frac{a}{a+b} \frac{F^{+}(v ; a+1, b)}{F^{+}(v ; a, b)}=\frac{a}{a+b} \frac{f(v ; a+1, b)}{f(v ; a, b)}+\frac{F^{+}(v ; a, b)}{f(v ; a, b)}
$$

Defining $\gamma=a+1$ and substituting for the density functions yields:

$$
\frac{a}{a+b} \frac{F^{+}(v ; a+1, b)}{F^{+}(v ; a, b)}=\frac{a}{a+b}\left(\frac{\frac{v^{\gamma-1}(1-v)^{b-1}}{B(\gamma, b)}}{\frac{v^{a-1}(1-v)^{b-1}}{B(a, b)}}\right)+\frac{F^{+}(v ; a, b)}{f(v ; a, b)}
$$

Expression (A9) reduces to:

$$
\frac{a}{a+b} \frac{F^{+}(v ; \gamma, b)}{F^{+}(v ; a, b)}=v+\frac{F^{+}(v ; a, b)}{f(v ; a, b)}
$$

Subtracting $v$ from both sides and multiplying by $1 / v$ yields the inverse elasticity pricing rule:

$$
\frac{\frac{a}{a+b} \frac{F^{+}(v ; a+1, b)}{F^{+}(v ; a, b)}-v}{v}=\frac{F^{+}(v ; a, b)}{v f(v ; a, b)}
$$

The left term is the mean surplus of one unit of supply relative to the price and the right term is the inverse price elasticity. 